\title{
Preexposure to conditioned and unconditioned stimuli in taste-aversion learning
}

\author{
STEPHEN W. KIEFER, JANICE A. PHILLIPS, and J. JAY BRAUN \\ Arizona State University, Tempe, Arizona 85281
}

\begin{abstract}
Four groups of rats were compared on the acquisition of a sucrose $(50 \mathrm{~g} / 1)$ aversion produced by sucrose-apomorphine $(20 \mathrm{mg} / \mathrm{kg})$ pairings. One group was preexposed to sucrose, another group was preexposed to drug-induced illness, and a third group was preexposed both to sucrose and to drug-induced illness. A fourth group was not preexposed to taste or drug. Results showed that preexposure to either the sucrose or the drug attenuated the acquisition of a sucrose aversion. Preexposure to both sucrose and drug produced a greater deficit in aversion learning than preexposure to either alone. It was concluded that taste preexposure and drug preexposure effects have independent and additive influences on taste-aversion learning.
\end{abstract}

Preexposure to a taste stimulus retards development of a learned aversion to that taste when it is paired later with drug-induced illness (Kalat, 1974; Kalat \& Rozin, 1973). Similarly, preexposure to an illness-inducing agent has been shown to produce deficits in taste-aversion conditioning (Brookshire \& Brackbill, 1976; Cannon, Berman, Baker, \& Atkinson, 1975; Riley, Jacobs, \& LoLordo, 1976). The following experiment examined the effect of taste (CS) preexposure and drug (US) preexposure on taste-aversion learning by combining these manipulations within a single experimental group. The experiment was designed to determine not only the individual effects of CS preexposure and US preexposure but also to detect a possible interaction between these factors.

\section{METHOD}

\section{Subjects}

The subjects were 40 naive male Long-Evans hooded rats, 150-160 days old, housed individually with food available ad lib. The rats remained in their home cages throughout the experiment and were removed only for injections and daily weight measures. A 9-h- (9:30 p.m. to 6:30 a.m.) dark/15-h-light cycle was maintained throughout the experiment.

\section{Procedure}

The present procedure was essentially the same as that originally employed by Braun, Slick, and Lorden (1972). Water was removed from the cages $24 \mathrm{~h}$ prior to the start of the experiment, and a watering schedule of one-bottle presentations for $15 \mathrm{~min}$ every $12 \mathrm{~h}(8: 30 \mathrm{a} . \mathrm{m}$. and 8:30 p.m.) was instituted and maintained throughout. Fluids were presented in $50-\mathrm{ml}$ calibrated centrifuge tubes fitted with one-hole stoppers and stainless steel drinking spouts. At the end of a 15-min drinking session, the tubes were removed and the amount of solution consumed was recorded to the nearest $.5 \mathrm{ml}$.

Distilled water was presented for 7 days to adapt the rats to the schedule. Four groups were formed $(n=10$ for each

This research was supported by U.S. Public Health Service Grant NS-16616 to J. Braun. Requests for reprints should be sent to J. Jay Braun, Department of Psychology, Arizona State University, Tempe, Arizona 85281. group), matched on the basis of mean water consumption over the last four presentations during adaptation.

Familiarization. Familiarization procedures began on the 8 th day. During familiarization, sucrose $(50 \mathrm{~g} / 1$ distilled water $)$ was presented to Groups SuD and SuP and salt $(9 \mathrm{~g} / 1$ distilled water) was presented to Groups StD and StP. These concentrations were chosen because they are highly palatable and clearly discriminable from water (Braun \& Kiefer, 1975). Each group was presented with its taste solution five times, with two water sessions interposed between each taste presentation.

Pretraining day. Distilled water was presented on the morning after the last familiarization session. Six hours later each rat in Groups SuD and StD was injected with apomorphine hydrochloride $(20 \mathrm{mg} / \mathrm{kg} \mathrm{IP})$. Rats in Groups SuP and StP were injected with an equivalent volume of physiological saline at this time. Distilled water was presented to all rats on the evening session of the pretraining day. The group designations reflect the familiarization and pretraining day manipulations: The first letters identify the solution to which the group was familiarized, sucrose $(\mathrm{Su})$ or salt $(\mathrm{St})$, and the last letter identifies the pretraining injection, either the drug apomorphine (D) or physiological saline $(\mathrm{P})$.

Training. On the morning following the pretraining day, all rats were presented with the $50-\mathrm{g} / 1$ sucrose solution and injected with apomorphine hydrochloride $(20 \mathrm{mg} / \mathrm{kg}$ IP) immediately upon termination of the drinking period. This initial training trial is referred to as Presentation IT. Following Presentation IT, each rat was assigned one of two sequences of fluid presentation: salt, distilled water, sucrose, or distilled water, salt, sucrose. These two sequences of fluid presentation were balanced within each group and were repeated three times. Injections of apomorphine followed each sucrose presentation.

The $50-\mathrm{g} / 1$ sucrose and $9-\mathrm{g} / 1$ salt solutions were made fresh daily from reagent-grade chemicals and distilled water and presented at room temperature $\left(24^{\circ} \mathrm{C}\right)$. Following a watering session, all tubes, rubber stoppers, and glassware were washed in hot Alconox solution, rinsed thoroughly with tap water, and finally rinsed with distilled water. The apomorphine hydrochloride was prepared $1 / 2 \mathrm{~h}$ before each sucrose session by dissolving 6-mg apomorphine hydrochloride tablets (Eli Lilly and Company) in distilled water $(3$ tablets/ $1 \mathrm{cc}$ ).

\section{RESULTS AND DISCUSSION}

\section{Familiarization}

Rats familiarized with sucrose consumed significantly more on the last familiarization trial than on the first: 
Familiarization Trial $1=15.3 \mathrm{ml}$, Familiarization Trial $5=19.5 \mathrm{ml}$ [paired $\mathrm{t}(19)=3.79, \mathrm{p}<.01$, twotailed]. Rats familiarized with salt did not show a significant change in salt consumption across familiarization trials (Familiarization Trial $1=15.7 \mathrm{ml}$, Trial $5=$ $15.7 \mathrm{ml}$ ). Thus, there was evidence of neophobia (Barnett, 1963) for only sucrose, and not salt, during familiarization.

\section{Pretraining}

The drug injection on the pretraining day iven to Groups SuD and StD did not significantly affect water consumption on the subsequent drinking session. The mean water consumption for the evening session was as follows: $\mathrm{SuD}, 12.9 \mathrm{ml}$; SuP, $11.9 \mathrm{ml}$; StD, $13.7 \mathrm{ml}$; StP, $13.0 \mathrm{ml}$. A 2 by 2 factorial analysis revealed no significant effect of drug injection or taste familiarization and no significant interaction between these two. Therefore, noncontingent drug injections did not produce obvious changes of subsequent water consumption when measured $6 \mathrm{~h}$ later.

\section{Training}

The results for the training phase of the experiment are shown in Figure 1. The mean consumption of sucrose, salt, and distilled water for each group is shown for three presentations.

Sucrose. The sucrose consumption on Presentation IT was analyzed in the same manner as was the evening water consumption on the pretraining day. On the initial training trial, there was no indication of a neophobic response to the sucrose. Rats for which sucrose was novel (Groups StD and StP) consumed essentially the same amount of sucrose as the rats for which sucrose was familiar. Pretraining injection was not a significant effect nor was the interaction of Familiar Taste by Pretraining Injection.

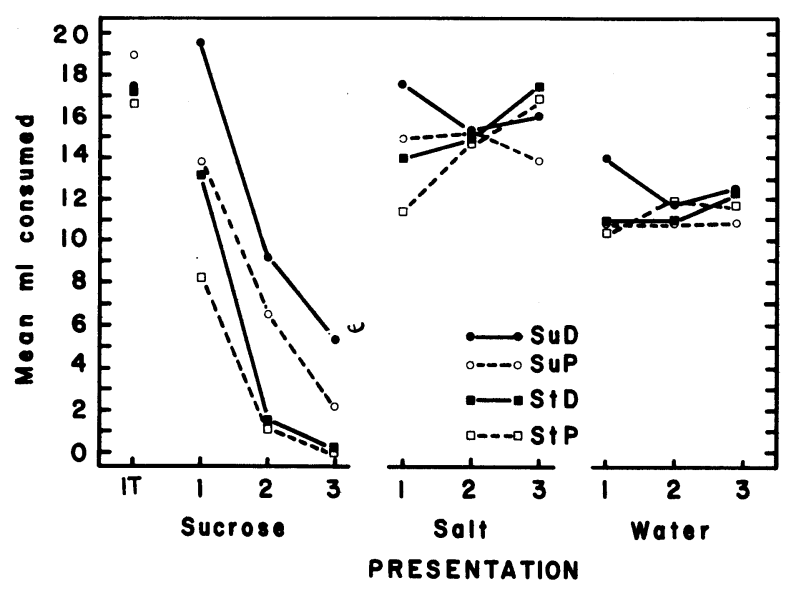

Figure 1. Mean milliliters consumed of sucrose, salt, and water by each group during the training phase. IT refers to the initial training trial.
Differences in sucrose consumption during Presentations 1 through 3 , as analyzed with a repeated measures analysis of variance, were significant. Both taste preexposure $[\mathrm{F}(1,36)=28.42, \mathrm{p}<.0001]$ and drug preexposure $[F(1,36)=7.85, p<.01]$ were significant main effects; there was no significant interaction of Taste Preexposure by Drug Preexposure. These results suggest that preexposure to sucrose or to drug-induced illness independently affected tasteaversion learning. Rats preexposed to sucrose showed a deficit in acquiring the sucrose aversion. Similarly, rats preexposed to illness also showed deficits in aversion acquisition.

A significant trial effect was found $[F(2,72)=204.34$, $\mathrm{p}<.0001]$ in addition to a marginally significant interaction of Trials by Taste Preexposure $[F(2,72)=$ 2.93, $\mathrm{p}=.058]$ and a significant Trials by Drug Preexposure interaction $[F(2,72)=7.08, p=.001]$. These significant interactions resulted from differences between the acquisition curves for the respective groups. Rats that were preexposed to either taste or drug did not reduce sucrose consumption as readily as rats given no prior experience with taste or drug.

Post hoc comparisons of sucrose consumption were made using the Scheffé Test for Multiple Comparisons. On Presentation 1, Groups StP and SuD differed significantly from each of the three other groups (ps $<.01)$. Group StP developed the greatest aversion, as evidenced by the reduced sucrose consumption on Presentation 1 . Group $\mathrm{SuD}$, on the other hand, did not acquire an apparent aversion to sucrose following the initial training trial. The groups preexposed to either sucrose (SuP) or to drug-induced illness (StD) displayed aversion acquisitions on Presentation 1 that were intermediate between the performances shown by Groups StP and $\mathrm{SuD}$. The group preexposed to the sucrose (SuP) and the group preexposed to the drug (StD) did display significant aversions to sucrose on Presentation 1: These groups consumed significantly less sucrose on Presentation 1 than on Presentation IT [paired ts $(9) \geqslant 3.05$, ps $<.02$, two-tailed]. Thus, on Presentation 1, preexposure to either the CS (taste) or the US (illness) attenuated the acquisition of a specific taste aversion and preexposure to both produced a greater attenuation than either alone. Comparisons of the total sucrose consumption across presentations showed that Group SuD drank significantly more sucrose than the other three groups, which further attests to this group's learning deficit $(\mathrm{p}<.01)$. Group StP drank significantly less sucrose than the other three groups $(p<.01)$.

Salt and water. The salt and water data were analyzed also with a repeated measures analysis of variance. The only significant effect found in salt consumption was the interaction of Trials by Taste Preexposure $[F(2,72)=$ $4.60, p=.013]$. The groups preexposed to salt increased their consumption of salt across trials, whereas the groups preexposed to sucrose drank slightly decreasing 
amounts across trials. No significant differences were found in the analysis of water consumption.

The present results confirm and extend previous reports of stimulus preexposure effects on taste-aversion learning. Preexposure to either a taste CS or a drug US attenuated aversion acquisition. A unique contribution of the present experiment is the evidence it provides for the independence and additivity of taste preexposure and drug preexposure effects. These results support the point made by Hearst and Jenkins (1974) that conditioning can be weakened by presenting CS-alone trials or by presenting US-alone trials. Similar independent and additive deleterious effects of CS preexposure and US preexposure have been demonstrated using a conditioned suppression paradigm (Baker, 1976, Experiment 2), which suggests that such effects are relatively ubiquitous in classical conditioning situations.

\section{REFERENCES}

BAKER. A. G. Learned irrelevance and learned helplessness: Rats learn that stimuli, reinforcers, and responses are uncorrelated. Journal of Experimental Psychology: Animal Behavior Processes, 1976, 2, 130-141.

BARnetT. S. A. The rat: A study in behaviour. Chicago: Aldine, 1963.

Braun. J. J.. \& Kiefer, S. W. Preference-aversion functions for basic taste stimuli in rats lacking gustatory neocortex. Bulletin of the Psychonomic Society, 1975, 6. 438-439. (Abstract)

Braun, J. J., Slick, T. B., \& Lorden, J. F. Involvement of gustatory neocortex in the learning of taste aversions. Physiology and Behavior, 1972, 9, 637-641.

Brookshire, K. H., \& BrackBILl, R. M. Formation and retention of conditioned taste aversions and UCS habituation. Bulletin of the Psychonomic Society, 1976, 7, 125-128.

Cannon, D. S., Berman, R. F., Baker, T. B., \& Atrinson, C. A. Effect of preconditioning unconditioned stimulus experience on learned taste aversions. Journal of Experimental Psychology: Animal Behavior Processes, 1975, 104, 270-284.

HEARST, E., \& JENKINS, H. M. Sign-tracking: The stimulus reinforcer relation and directed action. Austin, Tex: The Psychonomic Society, 1974.

Kalat, J. W. Taste salience depends on novelty, not concentration, in taste-aversion learning. Journal of Comparative and Physiological Psychology, 1974, 86, 47-50.

Kalat, J. W., \& Rozin, P. "Learned safety" as a mechanism in long-delay taste-aversion learning in rats. Journal of Comparative and Physiological Psychology, 1973, 83, 198-207.

Riley, A. L., JACOBS, W. J., \& LoLoRDo, V. M. Drug exposure and the acquisition and retention of a conditioned taste aversion. Journal of Comparative and Physiological Psychology, 1976, 90, 799-807.

(Received for publication May 23, 1977.) 